\title{
Immunogenicity of Recombinant Hepatitis B Vaccine Among Routinely Vaccinated Healthy and Chronically III Children in Assiut, Upper Egypt
}

\author{
Osama M. El-Asheer ${ }^{\mathrm{a}}$, Manal M. Darwish ${ }^{\mathrm{b}}$, Madleen A. Abdou ${ }^{\mathrm{c}}$, Khaled Saad $^{\mathrm{a}, \mathrm{d}}$
}

\begin{abstract}
Background: Egypt is considered a region of the intermediate prevalence of hepatitis B virus (HBV) infection (4.5\%). Seroprotection is assured when hepatitis B surface antibody (HBsAb) levels are $\geq 10 \mathrm{mIU} /$ $\mathrm{mL}$. Our study aimed to evaluate and compare the long-term immunogenicity and efficacy of the recombinant hepatitis B (HB) vaccine.
\end{abstract}

Methods: A cross-sectional study was done for children aged from 9 months to 15 years, receiving health care at Assiut University Children's Hospital, Assiut, Egypt in 3 months. HBsAb was quantitatively determined by enzyme-linked immune sorbent assay (ELISA).

Results: Seroprotection in infants less than 1 year was $89.7 \%$ with $55.2 \%$ having titer $>100 \mathrm{mIU} / \mathrm{mL}$ and this percent dropped to $64.4 \%$ after the first year of age with only $29 \%$ having titer $>100 \mathrm{mIU} /$ $\mathrm{mL}$. The overall protection percentage was $32.5 \%(>100 \mathrm{mIU} / \mathrm{mL})$, $34.7 \%$ of children showed levels between 10 and $100 \mathrm{mIU} / \mathrm{mL}$, while $32.8 \%$ were less than $10 \mathrm{mIU} / \mathrm{mL}$. Patients with diabetes mellitus were found to have the lowest seroprotective levels $(83.3 \%$ were not protected). Non-protective levels were also detected in patients with malnutrition (55.6\%), congenital heart diseases (43.2\%) and chronic liver diseases $(57.1 \%)$.

Conclusion: Our study shows failure to achieve satisfactory seroprotective levels for hepatitis B vaccine in both healthy and diseased children who adopted vaccination schedule in Upper Egypt. Booster dose in the second year of life is recommended for all children, particularly for those with diabetes millets, congenital heart disease and malnutrition.

Keywords: Immunogenicity; Hepatitis B vaccine; Children

\footnotetext{
Manuscript accepted for publication May 08, 2015

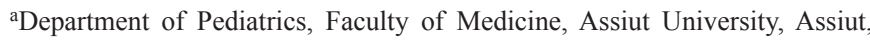
Egypt

bDepartment of Public Health \& Community Medicine, Faculty of Medicine, Assiut University, Assiut, Egypt

'Department of Clinical Pathology, Faculty of Medicine, Assiut University, Assiut, Egypt

${ }^{\mathrm{d} C}$ Corresponding Author: Khaled Saad, Faculty of Medicine, University of Assiut, Assiut 71516, Egypt. Email: Khaled.ali@med.au.edu.eg
}

doi: http://dx.doi.org/10.14740/gr636e

\section{Introduction}

Hepatitis B virus (HBV) infection is the most widespread chronic infectious disease worldwide. Nearly one-third of the world's population has serological evidence of past or present infection with HBV. An estimated 400 million persons worldwide are chronically infected with HBV. The global prevalence of HBV varies widely from low $(<2 \%$ as in Western Europe, North America and Japan) to high ( $>8 \%$ as in Africa, Southeast Asia and China). HBV infection is a major health problem in Egypt. Egypt is considered to be a region of intermediate prevalence for HBV infection with a reported figure of $4.5 \%$. Nearly 2 - 3 million Egyptians are chronic carriers of $\operatorname{HBV}[1,2]$.

Infection of infants and young children with HBV represents an important health hazard, since the younger the age at which the infection is acquired, the greater the predisposition to the carrier state, chronic liver disease and subsequent development of cirrhosis and hepatocellular carcinoma $[1,2]$.

Safe and effective vaccines against hepatitis B (HB) infection have been available since 1982. The World Health Organization (WHO) recommended universal vaccination against HB; this recommendation had been applied in Egypt. A compulsory vaccination program against $\mathrm{HBV}$ among infants was started in Egypt in 1992 using a yeast recombinant DNA vaccine $(10 \mu \mathrm{g})$ and with a schedule of 2, 4 and 6 months in age $[1,3,4]$.

The duration of protection after $\mathrm{HB}$ vaccination of infants is unknown, although many studies have found that after neonatal immunization with $\mathrm{HB}$ vaccine, a large proportion of the children, especially adolescents, exhibited waning immunity. Such decreased protection poses the risk of development infection [3-5].

Although advantageous for practical causes to incorporate HB vaccination into the schedule of the routine childhood immunization program, many authors have shown that short intervals of 1 or 2 months between the second and third doses of HB vaccine are accompanied by significantly lower levels of antibodies to HB surface antigen (anti-HBs) when compared to longer intervals of 4 months or more $[2,5,6]$.

The aim of our study is to evaluate and compare the longterm immunogenicity and efficacy of the recombinant HB vaccine administered to infants, according to the Egypt's National Immunization Program in both normal and chronically diseased children. 
Table 1. Distribution of Studied Children by Health Status

\begin{tabular}{lll}
\hline & Frequency & Percent $(\mathbf{\%})$ \\
\hline Normal & 139 & 53 \\
Renal failure & 30 & 11.4 \\
Diabetes mellitus & 12 & 4.6 \\
Congenital heart diseases & 37 & 14.1 \\
Malnutrition & 18 & 6.9 \\
Chronic liver diseases & 7 & 2.7 \\
Chronic hemolytic anemias & 19 & 7.3 \\
Total & 262 & 100 \\
\hline
\end{tabular}

\section{Patients and Methods}

\section{Study population}

This was a cross-sectional study. The participants were recruited from infants and children who were receiving health care at Assiut University Children's Hospital, Assiut, Egypt. The study was approved by the Researches and Ethical Review Committee, Faculty of Medicine, Assiut University. The date and dose intervals of $\mathrm{HB}$ vaccine were confirmed by checking the vaccination record written on the birth certificate of each child. The purpose of the study was carefully explained to the child's parents or guardians and informed consent to participate in the study was obtained before blood sampling.

A total of 262 children met the eligibility criteria which include: 1) age ranged from 9 months to 15 years; 2) born to hepatitis B surface antigen (HBsAg) negative mothers; 3) no history of prematurity; 4) had completed primary HB vaccination program according to the Egypt's National Immunization Program. A questionnaire was designed and administered to the parents or caretakers of the children to collect the basic demographic data (age, sex, etc.).

\section{Blood sampling and serological test}

Venous blood samples were collected from participants under standardized conditions. Samples were centrifuged $(3,000 \mathrm{~g}$ for $10 \mathrm{~min}$ ) and serum samples were stored in aliquots at -20 ${ }^{\circ} \mathrm{C}$ until analysis. Hepatitis B surface antibody (HBsAb) was quantitatively determined by enzyme-linked immune sorbent assay (ELISA) kit code number DEH02 from DIAKEY-Korea,
Table 2. Distribution of Seroprevalence of HBsAb Titer by Health Status

\begin{tabular}{lll}
\hline & Mean $(\mathrm{mIU} / \mathrm{mL})$ & SD \\
\hline Normal & 251.8 & 386.5 \\
Renal failure & 221.0 & 409.7 \\
Diabetes mellitus & 10.3 & 19.3 \\
Congenital heart disease & 53.3 & 85.6 \\
Malnutrition & 60.2 & 90.7 \\
Chronic liver disease & 46.1 & 81.6 \\
Chronic hemolytic anemias & 195.0 & 470.8 \\
Total & 186.4 & 350.2 \\
\hline
\end{tabular}

according to manufacturer's instructions.

The vaccine-induced antibody response was taken to be the anti-HBs level at 1 month following the third vaccine dose. Children with non-measurable (0.0) anti-HBs titers were considered seronegative (non-responders) and those with anti-HBs levels $<10 \mathrm{mIU} / \mathrm{mL}$ were considered to have an inadequate response. These two groups were not seroprotected. Those with anti-HBs levels $\geq 10 \mathrm{mIU} / \mathrm{mL}$ were considered to be seroprotected taking into consideration that those with anti-HBs levels between $10 \mathrm{mIU} / \mathrm{mL}$ and $100 \mathrm{mIU} / \mathrm{mL}$ were rated as having a low immune response needing a booster dose to have an adequate immune response and those with anti-HBs levels $>100$ $\mathrm{mIU} / \mathrm{mL}$ were rated as having a good immune response to the HB vaccine $[2,7,8]$.

\section{Statistical analysis}

Collected data were analyzed using SPSS, version 16. Descriptive analysis (mean and SD) was performed. Appropriate tests of significance were used to show statistical differences ( $t$-test, Chi-square, one-way ANOVA, etc.). To detect the relation between age and antibody titer, a correlation test was used. $\mathrm{P}$-value was considered significant if $<0.05$.

\section{Results}

Our study included 262 children (129 female, 49\%) aged from 9 months to 15 years. Eleven point one percent were under 1 year and $88.9 \%$ were from 1 to 15 years. All children were negative for HBsAg and had no history or clinical evidence of

Table 3. Distribution of Antibody Titer by Age

\begin{tabular}{llllll}
\multirow{2}{*}{ Age } & \multicolumn{3}{c}{ Antibody titer $(\mathbf{m I U} / \mathbf{m L})$} & Total & P-value* \\
\cline { 2 - 5 } & $<\mathbf{1 0}$ & $\mathbf{1 0}-<\mathbf{1 0 0}$ & $\mathbf{2 1 0 0}$ & & \multirow{2}{*}{$0.05^{*}$} \\
\hline One year or less (\%) & $3(10.3)$ & $10(34.5)$ & $16(55.2)$ & $29(100)$ & \\
More than one year (\%) & $83(35.6)$ & $81(34.8)$ & $69(29.6)$ & $233(100)$ & \\
Total (\%) & $86(32.8)$ & $91(34.7)$ & $85(32.5)$ & $262(100)$ & \\
\hline
\end{tabular}

*Significant. 
Table 4. Comparing 5-Year Age Groups Regarding Antibody Titer

\begin{tabular}{llll}
\hline & Mean difference & $\mathbf{9 5 \%}$ CI & P-value \\
\hline $\begin{array}{l}\text { Children }<5 \text { years versus } \\
\quad\end{array}$ & & & \\
$\quad$ 5 years to $<10$ years & 128.98 & $18.56-239.39$ & $0.022^{*}$ \\
$\quad \geq 10$ years & 169.45 & $67.92-270.97$ & $0.001^{*}$ \\
Children $\geq 5$ years to $<10$ years versus $\geq 10$ years & 40.47 & $-87.48-168.42$ & 0.534 \\
\hline
\end{tabular}

*Significant.

HBV infection. About half of the children in our study were clinically normal. Among the diseased children, congenital heart diseases were the most common (14.1\%), followed by renal failure $(11.4 \%)$, while chronic liver diseases came at the bottom of the list $(2.7 \%)$ (Table 1$)$. Table 2 shows the distribution of seroprevalence of HBsAb titer by health status. Seroprevalence of $\mathrm{HBsAb}$ titer was found to be below $100 \mathrm{mIU} / \mathrm{mL}$ in malnutrition, congenital heart disease, chronic liver disease and diabetes mellitus (in order from highest to lowest). More than half of our children aged less than 1 year had antibody (Ab) titer of $\geq 100 \mathrm{mIU} / \mathrm{mL}$ compared to only $29.6 \%$ of those aged more than 1 year with a statistical significant difference (Table 3). No significant difference was detected between males and females in the immunological response to HBV vaccine. There was a significant difference in the Ab titer between children aged less than 5 years and those aged from 5 to less than 10 years $(P=0.02)$. This difference became significantly higher when compared with those aged more than 10 years $(\mathrm{P}$ $=0.001)$. However, there was no statistical significant difference in the $\mathrm{Ab}$ titer between the older two groups $(\mathrm{P}=0.5)$ (Table 4). There was a highly significant difference in the $\mathrm{Ab}$ titer between normal children compared to diabetic children followed by children with congenital heart diseases than those with malnutrition (P value: $0.0001,0.017$ and 0.021 respectively) (Table 5). Figure 1 shows significant negative correlation between the age of the participating children and their $\mathrm{Ab}$ titer $(\mathrm{r}=-0.2 ; \mathrm{P}=0.0001)$.

\section{Discussion}

Evaluation of the long-term immunogenicity and the dura- tion of protection afforded by the recombinant $\mathrm{HB}$ vaccine are limited and incomprehensible. In addition, studies have not clearly established the need as booster doses have a better immunogenic response. Both host and immunization factors affect the immunogenic response to $\mathrm{HB}$ vaccine and consequently can influence the duration of immunity $[2,8,9]$. Age, weight, immune competence and genetics are considered as important host factors. Vaccine-related factors such as storage, transportation, cold chain, dose, site of immunization and vaccination schedule are considered as very important factors in immunogenicity of the vaccine [7-11]. Since the introduction of recombinant $\mathrm{HB}$ vaccine in 1980s, multiple studies were done to determine the antibody seroprotective levels (immune threshold) that varied among studies and they concluded that anti-HBs levels $<10 \mathrm{mIU} / \mathrm{mL}$ were considered to have inadequate immune response and need to be revaccinated, those with levels between 10 and $100 \mathrm{mIU} / \mathrm{mL}$ have low immune response and need booster dose of the vaccine and those with anti-HB levels $>100 \mathrm{mIU} / \mathrm{mL}$ were rated as having good immune response [2, 7-15].

Our study is the first study in Upper Egypt to assess the long-term immunogenicity and efficacy of the recombinant $\mathrm{HB}$ vaccine in both normal and chronically diseased children aged 9 months to 15 years. In the present study the overall seroprotection in infants less than 1 year was $89.7 \%$ with $55.2 \%$ having titer $>100 \mathrm{mIU} / \mathrm{mL}$ and this percent dropped to $64.4 \%$ after the first year of age with only $29.6 \%$ having titer $>100$ $\mathrm{mIU} / \mathrm{mL}$. The overall protection in our study was $32.5 \%$ ( $>$ $100 \mathrm{IU} / \mathrm{mL}$ ), 34.7\% show levels between 10 and $100 \mathrm{mIU} / \mathrm{mL}$ while $32.8 \%$ were $<10 \mathrm{mIU} / \mathrm{mL}$.

Many studies have investigated the long-term immunogenicity and efficacy of the recombinant $\mathrm{HB}$ vaccine in differ-

Table 5. Comparing Antibody Titer Between Normal Children and Others With Different Diseases

\begin{tabular}{|c|c|c|c|c|c|}
\hline & & Antibody titer (mI & & Totol $\mathbf{N}$ & D nuluo \\
\hline & $<10, \mathrm{~N}(\%)$ & $10-<100, \mathrm{~N}(\%)$ & $\geq 100, N(\%)$ & lotal, N (\%) & P-value \\
\hline Normal & $34(24.5)$ & $46(33.1)$ & $59(42.4)$ & $139(100)$ & \\
\hline Renal failure & $8(26.7)$ & $14(46.7)$ & $8(26.7)$ & $30(100)$ & 0.237 \\
\hline Diabetes mellitus & $10(83.3)$ & $2(16.7)$ & $0(0.0)$ & $12(100)$ & $0.0001 *$ \\
\hline Congenital heart disease & $16(43.2)$ & $14(37.8)$ & $7(18.9)$ & $37(100)$ & $0.017^{*}$ \\
\hline Malnutrition & $10(55.6)$ & $3(16.7)$ & $5(27.8)$ & $18(100)$ & $0.021 *$ \\
\hline Chronic liver disease & $4(57.1)$ & $2(28.6)$ & $1(14.3)$ & $7(100)$ & $0.031^{*}$ \\
\hline Chronic hemolytic anemia & $4(21.1)$ & $10(52.6)$ & $5(26.3)$ & $19(100)$ & 0.228 \\
\hline
\end{tabular}

*Significant. 


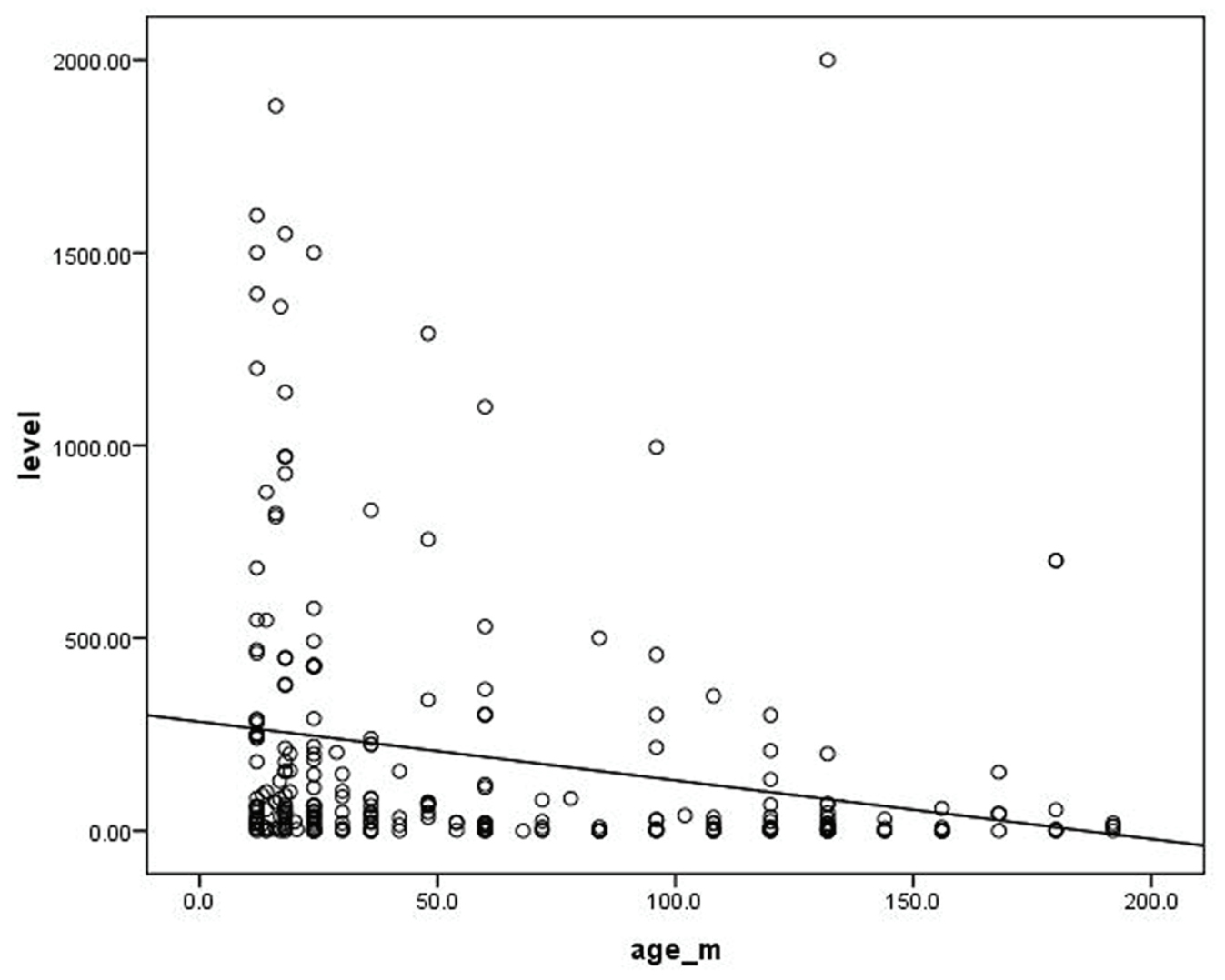

Figure 1. Correlation between age (in months) of the participating children and their $A b$ titer $(r=-0.2 ; P=0.0001)$.

ent countries. In agreement of the present study, two Egyptian studies showed seroprotection rates ranged from $39 \%$ [2] to $67 \%$ [11]. Norouzirad et al [12] reported seroprotection rates $90 \%$ among vaccinated Iranian children and $48.9 \%$ among adolescents. They observed declining titer of anti-HBsAb levels from $272.3 \mathrm{IU} / \mathrm{L}$ to $94.1 \mathrm{IU} / \mathrm{L}$ in 1 and 18 -year-old population, respectively. Similar to our results, they found a significant negative correlation between age and anti-HBsAb levels [12]. Aghakhani et al [13] reported seroprotection rates $65 \%$ in children 1 year after vaccination, and in $30 \%, 29 \%$ and $24 \%$ in 5, 10 and 15 years after vaccination, respectively. Other reports from Iran [10, 14-16] reported seroprotection rates from $29 \%$ to $75 \%$ among vaccinated Iranian children 5 - 10 years after vaccination. In Brazil, Livramento et al [17] reported seroprotection rate (anti-HBs $\geq 10 \mathrm{mIU} / \mathrm{mL}$ ) in $49.9 \%$ of individuals aged 10 - 15 years old. Other Brazilian study showed seroprotection in $58.9 \%$ of the studied population [18]. Alfaleh et al [19] reported that $38 \%$ of Saudi school students between the ages of 16 and 18 years showed protective anti-HBs titers. Other researchers reported lower levels of seroprotection among vaccinated children; Petersen et al [20] found that only $12.5 \%$ and Seto et al [9] found that $19 \%$ of the children had protective levels of anti-HBs antibodies. Marked drop in the antibody levels in our study from $89.7 \%$ in infants less than 1 year to $64.4 \%$ in those more than 1 year and decrease of antibody level over time that was also observed by previous reports $[2,9,11,12,18,20]$, from these data we concluded that rapid drop in the seroprotective levels over time recommended a booster dose of HBV.

Contrary to our data many studies from the developed countries reported the absence of breakthrough infection among the vaccinated population 20 years after vaccination. They also indicate a marked decline of acute HBV infection since the introduction of a mass vaccination program for infants. They concluded that there is no need for a booster dose of vaccine until individuals reach the age of 20 years [21-25].

In the present study, patients with diabetes mellitus were found to have the lowest seroprotective levels where $83.3 \%$ of diabetic children were non-protected and this was explained by Taheri et al [26], who stated that patients with diabetes mellitus have decreased vaccination response rates compared with healthy controls and this is due to a lower degree in antigen presentation and lower T-cell function. Patients with malnutrition showed non-protective levels in $55.6 \%$ whereas $16.7 \%$ showed low protective levels needing a booster dose and this may be due to the lower immunoglobulin levels and lower antibody levels in these children. Patients with congenital heart diseases showed non-protective levels in $43.2 \%$. Impaired humeral and cell-mediated immune response in patients with chronic renal failure [21] causing lower sero-convergence rate, low beak of antibody titer and quick decline of antibody levels can explain the results of our study as $26.7 \%$ were non-protect- 
ed and $46.7 \%$ show low protective levels and need a booster dose especially that those patients need repeated blood transfusion and more liable to get infection. More than half of patients with chronic liver diseases $(57.1 \%)$ were non-protected and $28.6 \%$ show low immune response. Hypoproteinemia and poor antibody formation in these patients can explain our results. The overall seroprotective levels were markedly lower in all diseased children compared to normal children with significant $\mathrm{P}$ value $<0.05$ in those with diabetes mellitus, congenital heart disease and malnutrition and this is suspected to be due to the more competent immune system in healthy children.

\section{Conclusion and recommendation}

Our study shows failure to achieve satisfactory seroprotective levels for HB vaccine in both healthy and diseased children who adopted vaccination schedule in Upper Egypt. Booster dose in the second year of life is recommended for all children, particularly for those with diabetes millets, congenital heart disease and malnutrition. Changing the route of administration (subcutaneous injection) or using 1,2 and 9 months schedule needs to be assessed and compared with the current route and schedule. We need randomized clinical trials to formulate future booster policies for preventing HB infection.

\section{Financial Disclosure}

The authors have no financial relationships relevant to this article to disclose.

\section{Conflict of Interest}

All authors declare no potential conflicts of interest with respect to the research, authorship, and/or publication of this article.

\section{Abbreviations}

Anti-HBs: antibody to hepatitis B surface antigen; ELISA: enzyme-linked immune sorbent assay; HB: hepatitis B; HBsAb: hepatitis B surface antibody; HBsAg: hepatitis B surface antigen; HBV: hepatitis B virus

\section{References}

1. Ismail S, Hafez HA, Darweesh SK, Kamal KH, Esmat G. Virologic response and breakthrough in chronic hepatitis B Egyptian patients receiving lamivudine therapy. Ann Gastroenterol. 2014;27(4):380-386.

2. Shaaban FA, Hassanin AI, Samy SM, Salama SI, Said ZN. Long-term immunity to hepatitis B among a sample of fully vaccinated children in Cairo, Egypt. East Mediterr Health J. 2007;13(4):750-757.
3. Agladioglu S, Beyazova U, Camurdan AD, Sahin F, Atak A. Immunogenicity of recombinant hepatitis $B$ vaccine: comparison of two different vaccination schedules. Infection. 2010;38(4):269-273.

4. Demirjian A, Levy O. Safety and efficacy of neonatal vaccination. Eur J Immunol. 2009;39(1):36-46.

5. Lu CY, Chiang BL, Chi WK, Chang MH, Ni YH, Hsu $\mathrm{HM}$, Twu SJ, et al. Waning immunity to plasma-derived hepatitis B vaccine and the need for boosters 15 years after neonatal vaccination. Hepatology. 2004;40(6):14151420 .

6. American Academy of Pediatrics Committee on Infectious Diseases: Universal hepatitis B immunization. Pediatrics. 1992;89(4 Pt 2):795-800.

7. Yu AS, Cheung RC, Keeffe EB. Hepatitis B vaccines. Clin Liver Dis. 2004;8(2):283-300.

8. Poland GA, Jacobson RM. Clinical practice: prevention of hepatitis B with the hepatitis B vaccine. N Engl J Med. 2004;351(27):2832-2838.

9. Seto D, West DJ, Ioli VA. Persistence of antibody and immunologic memory in children immunized with hepatitis B vaccine at birth. Pediatr Infect Dis J. 2002;21(8):793795.

10. Yazdanpanah B, Safari M, Yazdanpanah S. Persistence of HBV Vaccine's Protection and Response to Hepatitis B Booster Immunization in 5- to 7-Year-Old Children in the Kohgiloyeh and Boyerahmad Province, Iran. Hepat Mon. 2010;10(1):17-21.

11. Reda AA, Arafa MA, Youssry AA, Wandan EH, Ab de Ati M, Daebees H. Epidemiologic evaluation of the immunity against hepatitis B in Alexandria, Egypt. Eur J Epidemiol. 2003;18(10):1007-1011.

12. Norouzirad R, Shakurnia AH, Assarehzadegan MA, Serajian A, Khabazkhoob M. Serum levels of anti-hepatitis B surface antibody among vaccinated population aged 1 to 18 years in ahvaz city southwest of iran. Hepat Mon. 2014;14(1):e13625.

13. Aghakhani A, Banifazl M, Izadi N, McFarland W, Sofian M, Khadem-Sadegh A, Pournasiri Z, et al. Persistence of antibody to hepatitis B surface antigen among vaccinated children in a low hepatitis B virus endemic area. World J Pediatr. 2011;7(4):358-360.

14. Jafarzadeh A, Montazerifar SJ. Persistence of anti-HBs antibody and immunological memory in children vaccinated with hepatitis B vaccine at birth. J Ayub Med Coll Abbottabad. 2006;18(4):4-9.

15. Shamsizadeh A, Makvandi M, Shoshtari G. Prevalence of anti hepatitis B surface antibody among children in Ahvaz, Iran, five years after vaccination. J J Microbiol. 2011;4(1):49-54.

16. Javad Hosseini SM, Ranjbar R, Abolghasemi H, Turkaman M. Evaluation of the Level of HBV Antibody Titer after HBV Vaccination among Children in Tehran, Iran. Hepat Mon. 2009;9(2):150-153.

17. Livramento A, Cordova CM, Scaraveli NG, Tonial GC, Spada C, Treitinger A. Anti-HBs levels among children and adolescents with complete immunization schedule against hepatitis B virus. A cross-sectional study in Blumenau, State of Santa Catarina, Brazil, 2007-2008. Rev 
Soc Bras Med Trop. 2011;44(4):412-415.

18. Voigt AR, Strazer Neto M, Spada C, Treitinger A. Seroprevalence of hepatitis $\mathrm{B}$ and hepatitis $\mathrm{C}$ markers among children and adolescents in the south Brazilian region: metropolitan area of Florianopolis, Santa Catarina. Braz J Infect Dis. 2010;14(1):60-65.

19. Alfaleh F, Alshehri S, Alansari S, Aljeffri M, Almazrou Y, Shaffi A, Abdo AA. Long-term protection of hepatitis B vaccine 18 years after vaccination. J Infect. 2008;57(5):404-409.

20. Petersen KM, Bulkow LR, McMahon BJ, Zanis C, Getty M, Peters H, Parkinson AJ. Duration of hepatitis B immunity in low risk children receiving hepatitis $\mathrm{B}$ vaccinations from birth. Pediatr Infect Dis J. 2004;23(7):650-655.

21. Zanetti AR, Mariano A, Romano L, D'Amelio R, Chironna M, Coppola RC, Cuccia M, et al. Long-term immunogenicity of hepatitis B vaccination and policy for booster: an Italian multicentre study. Lancet. 2005;366(9494):13791384.

22. Banatvala J, Van Damme P, Oehen S. Lifelong protection against hepatitis B: the role of vaccine immunogenicity in immune memory. Vaccine. 2000;19(7-8):877-885.

23. Roznovsky L, Orsagova I, Kloudova A, Tvrdik J, Kabieszova L, Lochman I, Mrazek J, et al. Long-term protection against hepatitis $\mathrm{B}$ after newborn vaccination: 20 -year follow-up. Infection. 2010;38(5):395-400.

24. McMahon BJ, Dentinger CM, Bruden D, Zanis C, Peters H, Hurlburt D, Bulkow L, et al. Antibody levels and protection after hepatitis B vaccine: results of a 22-year follow-up study and response to a booster dose. J Infect Dis. 2009;200(9):1390-1396.

25. Gabbuti A, Romano L, Blanc P, Meacci F, Amendola A, Mele A, Mazzotta F, et al. Long-term immunogenicity of hepatitis B vaccination in a cohort of Italian healthy adolescents. Vaccine. 2007;25(16):3129-3132.

26. Taheri S, Shahidi S, Moghtaderi J, Seirafian S, Emami A, Eftekhari S. Response rate to Hepatitis B vaccination in patients with chronic renal failure and end-stage-renal-disease: Influence of diabetes mellitus. Journal of Research in Medical Sciences. 2005;10:384-390. 